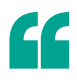

...improved response of patients to radiotherapy after chemical castration...

\title{
PROSTATE CANCER
}

\section{Castration impairs DNA repair}

Repair of DNA damage - in the form of DNA double-strand breaks (DSBs) - induced by radiotherapy is impaired by neoadjuvant castration therapy, according to new research published in Science Translational Medicine.

In many treatment centres, the combination of neoadjuvant castration with radiotherapy has become the standard of care for men with intermediate-risk or high-risk prostate cancer, and that chemical castration improves patient response to radiotherapy is recognized. Tarish and colleagues investigated the biological mechanism by which this effect occurs.

In this study, 48 men with localized prostate cancer who were eligible for curative radiotherapy were enrolled and assigned to one of two treatment arms; 25 men were allocated to receive neoadjuvant chemical castration with leuprorelin before undergoing external beam radiotherapy (arm one) and 23 men received radiotherapy for 5 days consecutively, then neoadjuvant chemical castration with leuprorelin followed by an increased dose of radiotherapy (arm two). Prostate tissue biopsy specimens were obtained from all patients before treatment commenced. In arm one, a second biopsy was taken before radiotherapy commenced and then a third obtained $3 \mathrm{~h}$ after the fifth fraction of radiation. In arm two, a second biopsy was taken $3 \mathrm{~h}$ after the fifth radiation fraction, before starting castration therapy and a third after chemical castration had been received.

In both treatment arms, castration therapy significantly reduced levels of $\mathrm{Ku} 70$ - a protein that is involved in DSB repair via nonhomologous end joining (NHEJ) - regardless of whether patients had received previous radiotherapy $(P<0.001)$. After receiving radiotherapy, a significant increase in intranuclear $\mathrm{Ku} 70$ was observed in prostate tissue from patients in arm two $(P=0.014)$, which was probably caused by radiotherapy-induced DSB repair. Upregulation of Ku70 was not observed in patients who had only received chemical castration.

To assess the affect of neoadjuvant castration on DSB repair, the expression and localization of 53BP1 and phosphorylated $\gamma$-H2AX - two commonly used markers of DSBs - were analysed using immunofluorescent staining. Radiotherapy induced a significant

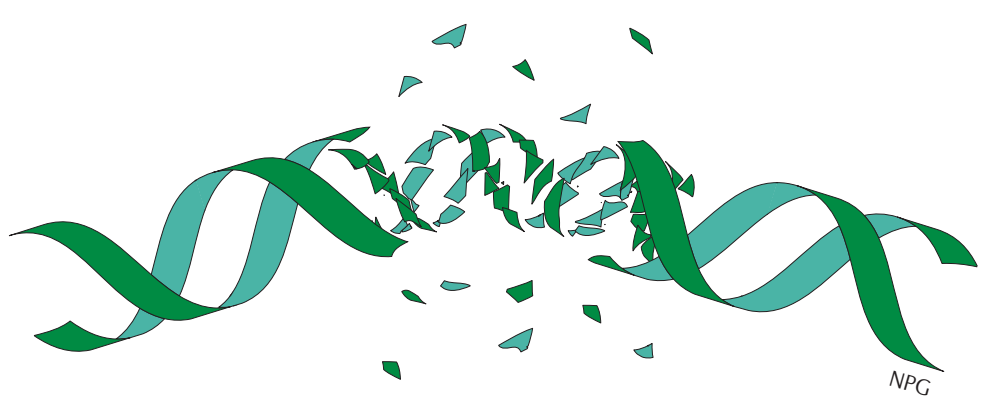

increase in 53BP1 and phosphorylated $\gamma$-H2AX foci $(P<0.001)$, which also colocalized. Castration alone did not increase DSBs and induced a significant reduction in intracellular 53BP1 in both arms $(P<0.050)$, indicating that the androgen receptor might be involved in 53BP1 expression. A significant increase in radiotherapy-induced $\gamma-\mathrm{H} 2 \mathrm{AX}$ was observed in patients in arm one, indicating that the number of DSBs was increased in these men.

The observed correlation between reduced Ku70 levels and increased DSB after chemical castration indicated that impairment of NHEJ might explain the decreased DSB repair and improved patient survival after radiotherapy with neoadjuvant castration. Ku70 is part of a heterodimer that recruits DNA-dependent protein kinase (DNA-PK) to DNA ends, and autophosphorylation of $\mathrm{Ser}^{2056}$ on DNA-PK - which is needed to complete NHEJ occurs. Radiotherapy increased phosphorylated DNA-PK, but neoadjuvant castration treatment before radiotherapy abolished this effect.

This study indicates that the improved response of patients to radiotherapy after chemical castration is explained by the impairment of NHEJ repair of DSBs by castration in prostate cancer.

Louise Stone

ORIGINAL ARTICLE Tarish, F. L. et al. Castration radiosensitizes prostate cancer tissue by impairing DNA double-strand break repair. Sci. Transl. Med.7, 312re11 (2015) 